\title{
Where do the Leads Lead? Severe Tricuspid Stenosis and Infection Secondary to Retained Leads
}

\author{
Harold P Rivner ${ }^{1 *}$, Mohammed S Ezuddin², Gary H Danton² and Roger Gr Carrillo ${ }^{3}$ \\ ${ }^{1}$ Department of Medicine, University of Miami Miller School of Medicine, USA \\ ${ }^{2}$ Department of Diagnostic Radiology, University of Miami Miller School of Medicine, USA \\ ${ }^{3}$ Department of Surgery, University of Miami Miller School of Medicine, USA
}

Submission: November 22, 2017; Published: December 14, 2017

*Corresponding author: Harold P Rivner, Department of Medicine, University of Miami Miller School of Medicine and Jackson Memorial Hospital, Email: Harold.rivner@jhsmiami.org

\section{Case Report}

A 58-year-old man was admitted to the hospital with asymptomatic hypertensive urgency. His medical history was significant for heart failure and complete heart block with a dual chamber pacemaker firstimplanted in 1985. Although he had more leads added over time, his previous leads were never removed. Examination revealed a left-sided generator and an old right-sided device with purulent eroded wires. He also had mild pedal edema. A chest radiograph (Figure 1A) demonstrated a complicated

lead arrangement with wires connecting the generator on the left and the old generator pocket site on the right, an atrial lead (yellow arrow) in the correct position, and multiple ventricular leads (green and red arrows) passing through the tricuspid area (oval). Gated chest computed tomography showed a misplaced, abandoned lead piercing one of the tricuspid leaflets (Red arrow in Figures 1B \& 1C). Echocardiogram shows resultant severe tricuspid stenosis with a mean pressure gradient of $11 \mathrm{mmHg}$ and a maximum velocity of $2.1 \mathrm{~m} / \mathrm{sec}$ through the valve (Video 1 ).

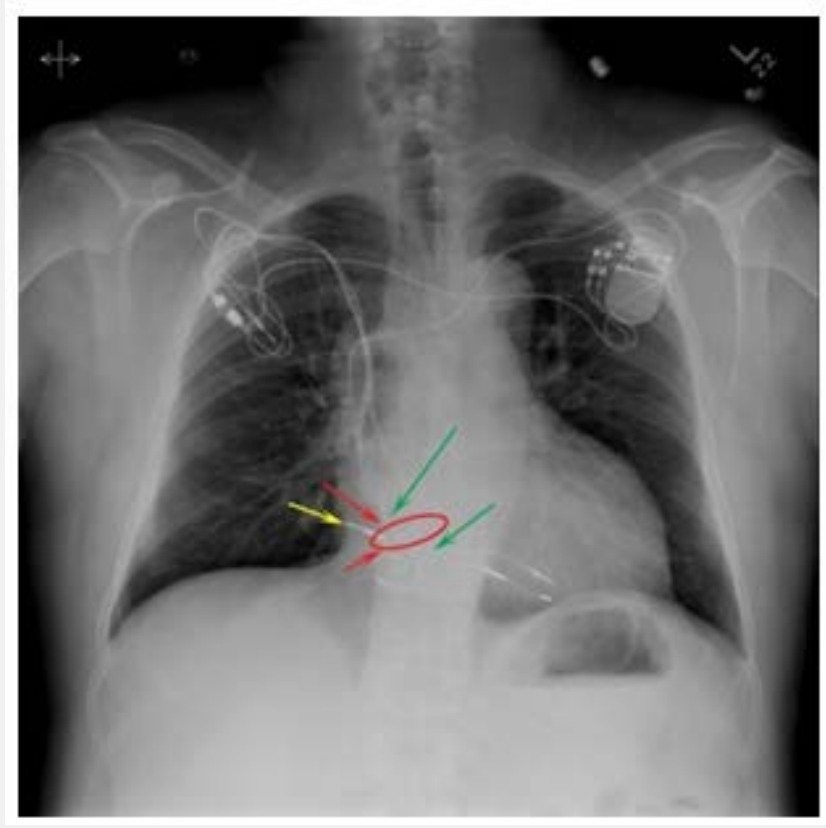

Figure 1: Chest Radiographs and Computed Tomography (CT). 1A: Chest radiograph demonstrating a complicated lead arrangement with wires connecting the generator and the old generator pocket site on the right, an atrial lead (yellow arrow) in the correct position, and multiple ventricular leads (green and red arrows) passing through the tricuspid area (oval). 


\section{Journal of Cardiology \& Cardiovascular Therapy}

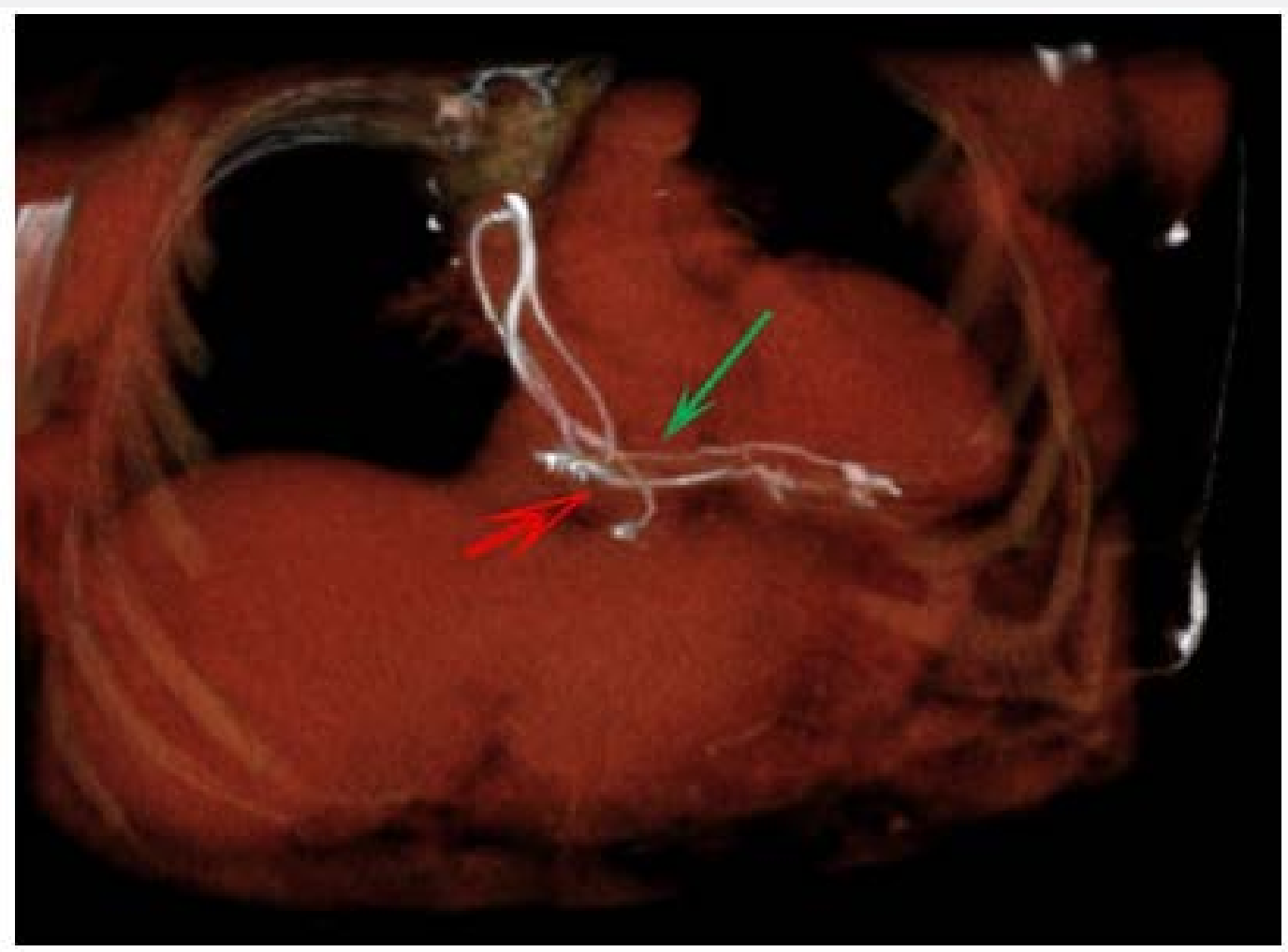

Figure 1B: Three-dimensional reconstruction of gated CT showing course of leads. The green arrow marks the region of the normal lead and the red arrow shows the anomalously placed lead.



Figure 1C: Cross sectional CT showing course of leads through the tricuspid valve. The two arrows approximate the region of the tricuspid valve. The green arrow shows the region where the misplaced lead pierces the tricuspid valve. The black arrow demarcates the region of the normal valve and the normal course of the ventricular lead. 


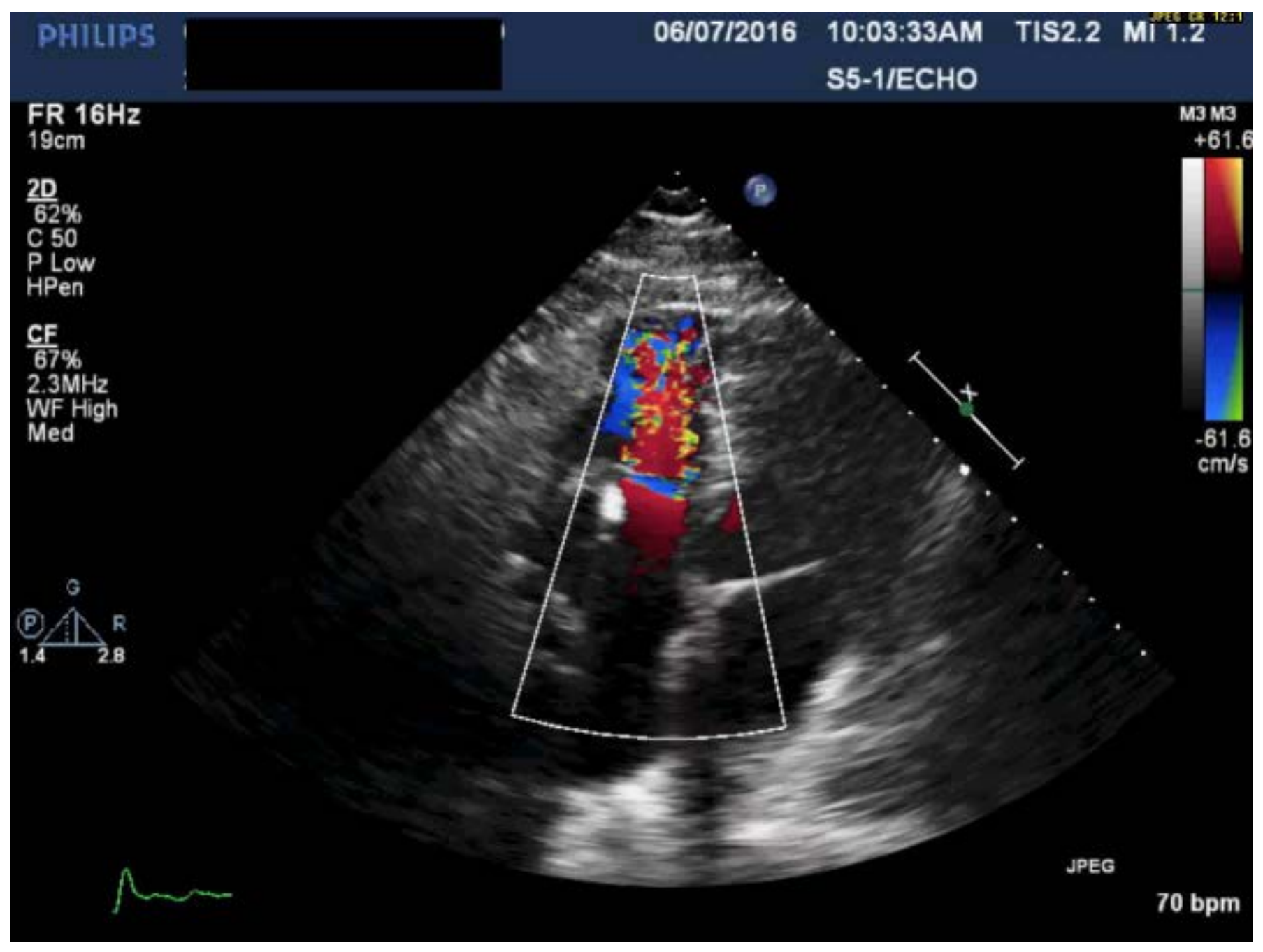

Video 1: Transthoracic Echocardiogram -Four chamber apical view shows severe tricuspid stenosis with stenosis with a mean pressure gradient of $11 \mathrm{mmHg}$ and a maximum velocity of $2.1 \mathrm{~m} / \mathrm{sec}$ through the valve.

Due to concern for lead infection and future development of right-sided heart failure from tricuspid stenosis, lead extraction was attempted. Cultures from the device pocket were positive for Staph epidermidis confirming the infection. Unfortunately, due to their age, fragmentation, and complexity, the leads were only partially removed and his tricuspid valve remained stenotic.

This case adds to a small but growing number of cases of traumatic tricuspid stenosis caused by cardiac device leads [1]. Furthermore, it illustrates how excess retained leads can serve as a nidus for infection. These images illustrate the danger and significant morbidity that excessive leads can cause.
Consequently, the images should serve as a reminder to not simply abandon old cardiac leads but to always weigh the risk of lead extraction with the potential significant complications from retained leads [2].

\section{References}

1. Rosenberg Y, Myatt JP, Feldman M, Carpenter AJ, Bauch T, et al. (2010) Down to the wire: tricuspid stenosis in the setting of multiple pacing leads. Pacing Clin Electrophysiol 33(5): 49-52.

2. Maytin M, Henrikson CA, Epstein LM (2010) Lead extraction is preferred for lead revisions and system upgrades: When less is more. Circ Arrhythmia Electrophysiol 3(4): 413-424. 